ARTICULO ORIGINAL

\title{
Etapas del modelo conductual de los estilos de vida propuestos por las guías alimentarias del Paraguay en adultos
}

\section{Stages of the behavioral model of life styles in adults proposed by food guides of Paraguay}

\author{
* Morínigo Martínez M, Samudio M
}

Instituto de Investigaciones en Ciencias de la Salud. Universidad Nacional de Asunción, Paraguay

\section{RESUMEN}

Las etapas del modelo conductual de las personas describen el cumplimiento o no de las recomendaciones para una vida sana. Por ello, se planteó una investigación observacional descriptiva cuyo objetivo fue evaluar las etapas del modelo conductual de los estilos de vida propuestos por las Guías Alimentarias del Paraguay en adultos que residen en el barrio Salvador del Mundo de Asunción. Las variables de interés fueron las etapas de cambio, medidas a partir del modelo de Prochaska, para el consumo de sal, ingesta de bebidas alcohólicas, actividad física y manipulación de alimentos, tres de ellas están relacionadas al no cumplimiento y dos al cumplimiento de las recomendaciones. El 92,9\% (91/98) de los adultos no cumple con las recomendaciones de actividad física y el $71,4 \%$ (70/98) con las recomendaciones de consumo de sal. El 46,9\% (46/98) cumple con la recomendación para el consumo de bebidas alcohólicas, aunque el 74,5\% (73/98) refirió su consumo. Ningún encuestado cumple con las recomendaciones de lavado y manipulación de alimentos. Es preocupante el alto porcentaje de adultos que no cumple las recomendaciones, agravado por la no intención de cambio. Por lo tanto, se puede concluir que el objetivo inicial de las guías alimentarias del Paraguay de orientar a la población sobre los estilos de vida adecuados no se cumple. Debido a esto, se debe promover la revisión del medio de implementación de las mismas para alcanzar a un sector mayor de la población, y así lograr la adopción de mejores estilos de vida.

Palabras clave: modelo conductual, Guías Alimentarias del Paraguay, actividad física, consumo de sal, bebidas alcohólicas, higiene, manipulación de alimentos.

\section{ABSTRACT}

The stages of the behavioral model describe the compliance or not of the recommendations for a healthy life. Due to this, a descriptive observational study was proposed to evaluate the stages of the behavioral model of the life styles proposed by the Food Guides of Paraguay in adults living in the Salvador del Mundo neighborhood of Asunción. The variables were the stage of changes, measured from Prochaska's model, for salt consumption, alcohol intake, physical activity and food handling, three of them related to noncompliance and two to compliance of the recommendations. The $92.9 \%$ $(91 / 98)$ of the adults did not comply with the recommendations of physical activity and $71.4 \%(70 / 98)$ with the recommendations of salt intake. The $46.9 \%(46 / 98)$ complied with the recommendation of alcohol intake although it was observed that $74.5 \%(73 / 98)$ consumed some. None of the survey respondents complied with the recommendations of food cleaning and handling. It is worrying the high percentage of adults that did not comply with the recommendations of physical activity, worsened by the non-intention of changing. Therefore, it can be concluded that the initial objective of the food guides of Paraguay to orientate population about the adequate life styles is not complied. Due to

*Autor Correspondiente: Lic. Macarena Morínigo Martínez,

Email: mmorinigomartinez@hotmail.com

Fecha de recepción: julio 2014; Fecha de aceptación: diciembre 2014 
this, the revision of the implementation means or system should be encouraged to reach a larger sector of the population and thus, comply with the adoption of better life styles.

Keywords: behavioral model, Food Guides of Paraguay, physical activity, sal intake, alcohol intake, hygiene, food handling.

\section{INTRODUCCIÓN}

En el mundo, se puede advertir que los patrones alimentarios han dado un giro hacia conductas más occidentalizadas, caracterizadas por el consumo excesivo de alimentos en cuanto a energía, proteínas y grasas de origen animal. Esto sumado al alto porcentaje de sedentarismo y prácticas de estilo de vida inadecuadas, como el hábito de fumar y la ingesta excesiva de alcohol. Por todo lo anterior, surge la necesidad de incentivar la elaboración de materiales o guías alimentarias como estrategia para la promoción de la salud de los países (1).

La educación nutricional orienta sus recursos hacia el aprendizaje, adecuación y aceptación de los hábitos alimentarios saludables, que se formulan de acuerdo a la propia cultura alimentaria de cada país y a los conocimientos científicos en materia de nutrición. Consiste en la combinación de diferentes estrategias educativas, que motiven a la necesidad de cambio con el fin de desarrollar las habilidades que permitan una elección adecuada de alimentos y la adopción y mantenimiento de una conducta positiva en el tiempo, de tal manera a acercar a las personas a la salud y al bienestar alimentario (24).

James Prochaska (1979) propuso el modelo transteórico del cambio del comportamiento en salud que ofrece posibilidades para planear y ejecutar intervenciones a partir de las características específicas de las poblaciones a quienes están dirigidas las acciones. Detecta cinco etapas definidas: pre-contemplación, contemplación, preparación, acción y mantenimiento. Este modelo ha sido empleado en diferentes investigaciones e intervenciones sobre comportamientos ya reconocidos como de riesgo para la salud (5).

La Organización para la Alimentación y Agricultura y la Organización de la Salud (FAO/OMS) reconocen que, para contribuir a mejorar la calidad de vida de la población y prevenir las enfermedades relacionadas con la alimentación, la educación en nutrición a la población es primordial Sin embargo, previa a una implementación educativa es necesaria la identificación de las conductas. Las Guías Alimentarias Paraguay fueron elaboradas en el Instituto de Nutrición y Alimentación (INAN) y consta de 12 recomendaciones, 9 de las cuales están enfocadas a la alimentación y 4 a los estilos de vida. Están destinadas prevenir las enfermedades crónicas y degenerativas de la población $(6,7)$.

Este estudio se planteó para describir las etapas del modelo conductual de los estilos de vida (actividad física, consumo de sal, ingesta de bebidas alcohólicas e higiene de los alimentos) y las prácticas sobre manipulación e higiene de alimentos (lavado de las manos, lavar los utensilios, cocción de los alimentos, calentamiento de los alimentos y almacenamiento de alimentos) propuestas por las guías alimentarias del Paraguay en adultos que residen en el barrio Salvador del Mundo de Asunción.

\section{METODOLOGÍA}

El diseño de investigación fue observacional descriptivos de corte trasversal. Los sujetos de estudio seleccionados fueron adultos de 25 a 60 años de edad sanos, de ambos sexos, que residan en el Barrio Salvador del Mundo de Asunción.

Se midieron variables descriptivas como las socio-demográficas, el consumo de sal yodada, la ingesta de alcohol, tipo de consumidor de alcohol, la realización de ejercicio y práctica de higiene y manipulación de alimento (6).

Las variables de interés fueron el cumplimiento de las recomendaciones sobre estilos de vida y manipulación de alimentos propuestos por GAP: consumo de sal (hasta 1 cucharita por día, lo que corresponde a $5 \mathrm{~g} / \mathrm{d}$ ), el consumo de bebidas alcohólicas debe ser igual o 
menos de $168 \mathrm{~g} /$ semana (10 $1 / 2$ latas o $5 \quad 1 / 4$ botellas de cerveza por semana, o $17 \quad 1 / 2$ rayas de caña por semana o 14 copas de vino por semana) para hombre e igual o menos de $112 \mathrm{~g} /$ semana ( 7 latas 03 1/2 botellas de cerveza por semana, o 10 1/2 rayas de caña por semana o 10 1/2 copas de vino por semana) para mujeres. La recomendación para actividad física es de 30 minutos diarios y las recomendaciones para las prácticas higiénicas fueron el lavado de manos antes y después la manipulación de alimentos, antes de consumir los alimentos, lavar los alimentos con agua de la canilla o potabilizada, lavar los utensilios antes de utilizarlos y si lo hace con agua y jabón, utilizar utensilios según si sean crudos o cocidos, cocinar los alimentos hasta completar la cocción, recalentar las comidas cocidas hasta que estén bien calientes (hasta los $75^{\circ} \mathrm{C}$ ) y una vez cocidos los alimentos, no dejar fuera de la heladera por más de 2 horas.

Las etapas de cambio fueron clasificadas teniendo en cuenta las recomendaciones propuestas por las GAP: la ingesta de sal no debe superar los $5 \mathrm{~g}$ por día (1cucharita), el consumo de alcohol no debe ser superior a $168 \mathrm{~g} / \mathrm{semana}$ de etanol para hombres y 112 $\mathrm{g} / \mathrm{semana}$ de etanol para mujeres, la actividad física mínima debe ser de 30 minutos diarios y se debe cumplir con la totalidad de las práctica de higiene y manipulación de alimentos. En el caso que las personas no cumplieran las recomendación se indagó sobre si una vez conocidas las mismas en que tiempo cumplirían las mismas, clasificándose en: pre-contemplación, no tiene intención de cambiar y/o siente que no tiene que cambiar; contemplación, mayor conciencia sobre la necesidad de cambio (la persona piensa sobre el cambio, sin tomar acción o está indeciso); preparación, intención de tomar acción en el futuro cercano (0-3 meses). En caso que las personas cumplan con las recomendaciones, se indagó sobre el tiempo de cumplimiento, en forma de poder clasificarlas en: acción, cumple con las recomendaciones y las piensa mantener entre 3-6 meses; mantenimiento, cumple con las recomendaciones y piensa mantenerlas por 6 o más meses $(4,6)$.

Para la recolección de datos, se empleó una encuesta previamente validada. Se utilizó la entrevista como método o técnica de recolección de datos. Para el análisis de datos, se empleó el Software EfiInfo ${ }^{\circledR}$ versión 7.0. La investigación se realizó con la aprobación del comité científico-ético del Instituto de Investigación en Ciencias de la Salud (IICS) según el acta de aprobación No03/2012.

\section{RESULTADOS}

De la investigación participaron 98 personas, siendo el 53,1\% $(n=52)$ hombres y 46,9\% $(n=46)$ mujeres. El $51 \%(n=50)$ de la muestra estuvo conformada por jóvenes entre 20 a 34 años, 71,4\% refirió haber cursado el nivel secundario. (Tabla 1). El promedio de edad fue de $36 \pm 11$ años (IC95\% 34-38) y el promedio de escolaridad fue de $11,5 \pm 2,4$ años (IC95\% 11-12). 
Tabla 1. Datos demográficos de los encuestados. $n=98$

\begin{tabular}{llll}
\hline \multicolumn{2}{l}{ Datos demográficos } & $\mathbf{n}$ & $\mathbf{\%}$ \\
\hline \multirow{2}{*}{ Sexo } & Masculino & 52 & 53,1 \\
& Femenino & 46 & 46,9 \\
\multirow{2}{*}{ Rango de edad } & 20 a 34 años & 50 & 51,0 \\
& 35 a 49 años & 33 & 33,7 \\
\multirow{2}{*}{ Nivel de } & Mayores a 50 años & 15 & 15,3 \\
escolaridad & Primario & 7 & 7,1 \\
& Secundario & 70 & 71,4 \\
& Universitario & 21 & 21,4 \\
\hline
\end{tabular}

Según las etapas de Prochaska de las cinco etapas del cambio, tres hacen relación a la no realización de actividad física (pre-contemplación, contemplación y preparación), 91/92 (92,9\%), mientras que las dos restantes están relacionadas con la práctica habitual de actividad física, en cuanto a la duración de mínimo 30 minutos todos los días (7/98; $7,1 \%)$. En la presente investigación, se encontró que 91/98 no cumplían con las recomendaciones, de ellos 44\% (40/91) se encontraba en la etapa de pre-contemplación y contemplación (Tabla 2 ).

Tabla 2. Etapas de Prochaska de actividad física $(n=98)$

\begin{tabular}{|c|c|c|c|c|}
\hline \multicolumn{3}{|c|}{ Etapas de Prochaska para el cumplimiento de actividad física } & $\mathbf{n}$ & $\%$ \\
\hline \multirow{3}{*}{$\begin{array}{l}\text { No cumplimiento } \\
\text { de las } \\
\text { recomendaciones }\end{array}$} & $\begin{array}{l}\text { Pre- } \\
\text { contemplación }\end{array}$ & $\begin{array}{l}\text { No tiene intención de cumplir } \\
\text { con las recomendaciones }\end{array}$ & 40 & 44,0 \\
\hline & Contemplación & $\begin{array}{l}\text { Mayor conciencia sobre la } \\
\text { necesidad de cambio }\end{array}$ & 40 & 44,0 \\
\hline & Preparación & Intención de tomar acción & 11 & 12,0 \\
\hline Sub-Total $(n=91)$ & & & 91 & 92,9 \\
\hline $\begin{array}{l}\text { Cumplimiento de } \\
\text { las } \\
\text { recomendaciones }\end{array}$ & $\begin{array}{l}\text { Acción y/o } \\
\text { mantenimiento }\end{array}$ & $\begin{array}{l}\text { Cumple con las } \\
\text { recomendaciones }\end{array}$ & 7 & 100,0 \\
\hline Sub-Total $(n=7)$ & & & 7 & 7,1 \\
\hline
\end{tabular}

Según las etapas de Prochaska, de las 5 etapas del cambio, 3 se relacionan al no cumplimiento del agregado de sal, es decir el agregado de sal en cantidades mayores a 1 cucharadita $(70 / 82 ; 84,5 \%)$, mientras que las 2 etapas restantes están relacionadas con el consumo dentro de las recomendaciones, es decir, al no agregado de sal a las comidas o al agregado hasta 1 cucharadita de sal por día $(5 \mathrm{~g} / \mathrm{d})$. En la presente investigación, se encontró que 50/70 (71,5\%) no tenían intenciones de cumplir con las recomendaciones, por más que tenían el conocimiento de las recomendaciones, $15 / 70(21,4 \%)$ tenían conciencia que no cumplían con las recomendaciones, sin embargo, no tenían intenciones de cambios; mientras que 5/70 (7,1\%) tenían intenciones de cumplir con las recomendaciones en un corto tiempo (Tabla 3). 
Tabla 3. Etapas de Prochaska del agregado de sal en la comidas $(n=98)$

\begin{tabular}{|c|c|c|c|c|}
\hline \multicolumn{3}{|c|}{ Etapas de Prochaska para el consumo de sal } & $\mathbf{n}$ & $\%$ \\
\hline \multirow{3}{*}{$\begin{array}{l}\text { No cumplimiento de } \\
\text { las } \\
\text { recomendaciones }\end{array}$} & $\begin{array}{l}\text { Pre- } \\
\text { contemplación }\end{array}$ & $\begin{array}{l}\text { No tiene intención de cumplir } \\
\text { con las recomendaciones }\end{array}$ & 50 & 71,4 \\
\hline & Contemplación & $\begin{array}{l}\text { Mayor conciencia sobre la } \\
\text { necesidad de cambio }\end{array}$ & 15 & 21,4 \\
\hline & Preparación & Intención de tomar acción & 5 & 7,1 \\
\hline Sub-Total $(n=70)$ & & & 70 & 71,4 \\
\hline $\begin{array}{l}\text { Cumplimiento de las } \\
\text { recomendaciones }\end{array}$ & $\begin{array}{l}\text { Acción y/o } \\
\text { mantenimiento }\end{array}$ & $\begin{array}{l}\text { Cumple con las } \\
\text { recomendaciones }\end{array}$ & 28 & 100,0 \\
\hline Sub-Total $(n=28)$ & & & 28 & 28,6 \\
\hline
\end{tabular}

Según las etapas de Prochaska, de las 5 etapas del cambio, 3 se relacionan al no cumplimiento del consumo de alcohol $(46 / 98 ; 46,9 \%)$, mientras que las 2 etapas restantes están relacionadas con el consumo dentro de las recomendaciones, es decir, al no consumo de bebidas alcohólicas o al consumo dentro de las recomendaciones (52/52; $53,1 \%)$. En la presente investigación, se encontró que 36/46 (51,4\%) no tenían intenciones de cumplir con las recomendaciones, por más que tenían el conocimiento de las mismas, $7 / 46(10,0 \%)$ tenían mayor conciencia que no cumplían con las recomendaciones, sin embargo, tenían intenciones de cambios; mientras que 3/46 $(4,3 \%)$ tenían intenciones de cumplir con las recomendaciones en un corto tiempo (Tabla $4)$.

Tabla 4. Etapas de Prochaska de consumo de alcohol $(n=98)$

\begin{tabular}{|c|c|c|c|c|}
\hline \multicolumn{3}{|c|}{ Etapas de Prochaska para el consumo de bebidas alcohólicas } & \multirow{2}{*}{$\begin{array}{l}\mathbf{n} \\
36\end{array}$} & \multirow{2}{*}{$\begin{array}{l}\% \\
78,3\end{array}$} \\
\hline \multirow{3}{*}{$\begin{array}{l}\text { No cumplimiento de } \\
\text { las recomendaciones }\end{array}$} & Pre-contemplación & $\begin{array}{l}\text { No tiene intención de cumplir con } \\
\text { las recomendaciones }\end{array}$ & & \\
\hline & Contemplación & $\begin{array}{l}\text { Mayor conciencia sobre la } \\
\text { necesidad de cambio }\end{array}$ & 7 & 15,2 \\
\hline & Preparación & Intención de tomar acción & 3 & 6,5 \\
\hline Sub-Total $(n=46)$ & & & 46 & 46,9 \\
\hline $\begin{array}{l}\text { Cumplimiento de las } \\
\text { recomendaciones }\end{array}$ & $\begin{array}{l}\text { Acción } y / 0 \\
\text { mantenimiento }\end{array}$ & Cumple con las recomendaciones & 52 & 100,0 \\
\hline Sub-Total $(n=52)$ & & & 52 & 53,1 \\
\hline
\end{tabular}

Según las etapas de Prochaska, de las 5 etapas del cambio, 3 se relacionan al no cumplimiento de las recomendaciones de lavado y manipulación de alimentos, mientras que las 2 etapas restantes están relacionadas con el lavado de las manos antes y después de la manipulación de alimentos; el lavado de las manos antes de consumir los alimentos; el lavado de los alimentos con agua de la canilla o potabilizada, el lavado de los utensilios antes de utilizarlos y si lo hace agua y jabón; si utiliza utensilios según si sean crudos o cocidos; si cocina los alimentos hasta completar la cocción, si recalienta las comidas cocidas hasta que estén bien calientes y si no pasa más de 2 horas las comidas cocidas fuera de la heladera. Ninguno de los encuestados cumplía completamente con las recomendaciones, sin embargo, 68/98 (97,1\%) tenían intenciones de tomar acción (Tabla 5). 
Tabla 5. Etapas de Prochaska de lavado y manipulación de alimentos $(n=98)$

\begin{tabular}{|c|c|c|c|c|}
\hline \multicolumn{3}{|c|}{ Etapas de Prochaska para el lavado y manipulación de alimentos } & $\mathbf{n}$ & $\%$ \\
\hline \multirow{3}{*}{$\begin{array}{l}\text { No cumplimiento de las } \\
\text { recomendaciones }\end{array}$} & Pre-contemplación & $\begin{array}{l}\text { No tiene intención de cumplir } \\
\text { con las recomendaciones }\end{array}$ & 13 & 13,3 \\
\hline & Contemplación & $\begin{array}{l}\text { Mayor conciencia sobre la } \\
\text { necesidad de cambio }\end{array}$ & 17 & 17,3 \\
\hline & Preparación & Intención de tomar acción & 68 & 69,4 \\
\hline Sub-Total $(n=98)$ & & & 98 & 100,0 \\
\hline $\begin{array}{l}\text { Cumplimiento de las } \\
\text { recomendaciones }(n=0)\end{array}$ & 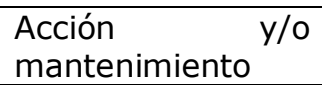 & $\begin{array}{l}\text { Cumple con } \\
\text { recomendaciones }\end{array}$ & 0 & 0,0 \\
\hline Sub-Total $(n=0)$ & & & $\mathbf{0}$ & 0,0 \\
\hline
\end{tabular}

\section{DISCUSIÓN}

Las enfermedades cardiovasculares, cáncer, diabetes, obesidad y otras enfermedades crónicas están aumentando rápidamente en todo el mundo (8). Este grupo de enfermedades comparte factores de riesgo comunes como el consumo de tabaco, sal y la inactividad física. Para la prevención y control de las Enfermedades no transmisibles (ENCT) se debe reducir los factores de riesgo asociados a esas enfermedades y se debe aplicar un enfoque integral que involucre a los sectores de salud, económico, educación, agricultura y planificación. La estrategia de menor costo, que tiene objetivos a corto y largo plazo, son las guías alimentarias $(4,9)$.

Las etapas del cambio de comportamiento en salud, propuesto por Prochaska, es uno de los modelos más ampliamente difundidos y aplicados en la actualidad para explicar, intervenir y evaluar las prácticas de comportamiento con efectos en la salud. Los resultados de la misma permiten planear y ejecutar intervenciones a partir de las características específicas de las poblaciones a quienes están dirigidas las acciones. El modelo detecta cinco etapas definidas: precontemplación, contemplación, preparación, acción y mantenimiento $(4,5,10)$.

La inactividad es causa de enfermedad y existe una relación dosis/respuesta entre actividad física y mortalidad global. Las personas que mantienen unos niveles razonables de actividad, tienen una menor probabilidad de padecer enfermedades crónicas o una muerte prematura. El consumo severo de bebidas alcohólicas es uno de los principales factores de riesgo relacionados con el estado de salud $(11,12)$, así mismo, el consumo excesivo de sal es riesgoso para el sistema cardiovascular.

En la presente investigación se encontró que el 32\% de los adultos encuestados realizaba actividad física, sin embargo, solo el $7,1 \%$ de los encuestados cumplía con las recomendaciones en cuanto a duración y frecuencia, tendencia que coincide con los reportado por Cabrera et al. En la presente investigación se encontró que el $44 \%$ no tiene intención de cambiar su comportamiento, mientras que en la investigación de Cabrera et al. este porcentaje fue de solo 19,4\%. (13). Dicha situación podría deberse a que Paraguay presenta uno de los índices mayores de sedentarismo, pues a nivel mundial, el sedentarismo asciende al $60 \%$, mientras que en Paraguay asciende a $74 \%$ de la población. En la presente investigación se encontró que el $46 \%$ de las personas eran consumidores severos de alcohol La misma tendencia fue reportada en la primera encuesta de factores de riesgo cardiovasculares del Paraguay, donde se encontró que el $50,9 \%$ de adultos mayores de 15 años consumía abusivamente alcohol. Solo el 4,3\% de los adultos con consumo severo tenían intención de reducir el consumo de alcohol. El $70 \%$ consumía más de 5 gramos de sal por día, de estos solo el 7,1\% de los encuestados que no cumplía con las recomendaciones refirieron tener intención de cambiar este hábito.

La insalubridad de los alimentos ha representado un problema de salud para el ser humano ya que los alimentos constituyen un medio para la transmisión de enfermedades 
alimentarias. En la presente investigación se encontró que ningún encuestado cumplía con las recomendaciones, sin embargo, el 97,1\% tenía intención de cambiar.

Se podría concluir que la población encuestada presenta un alto riesgo a enfermedades crónicas no trasmisibles, debido a la elevada presencia de sedentarismo, consumo elevado de sal y consumo de alcohol, así como el riesgo de enfermedades trasmitidas por alimentos por inadecuada manipulación de alimentos. Resultó llamativo que a pesar de las recomendaciones, la mayoría de los encuestados no tenía intención de cambiar sus estilos de vida, a pesar de conocer los beneficios de estilos de vida más saludables. Tras los 13 años de implementación de las Guías Alimentarias del Paraguay (14), no se observan cambios ni intención de cambios dentro de la población de estudio, resultados que deberían llamar la atención para cambiar las estrategias de promoción de las guías que se centran en puestos de atención de la salud, escuelas y otros, y muchas veces los adultos desconocen que existen estás herramientas de orientación. Además, la adopción de un estilo de vida está relacionado a diversos aspectos de la persona, como por ejemplo, gusto, situación laboral, sicológica, y/o familiar, entre otros por lo cual el cambio a estilos de vida más saludables no solo se vería con la educación nutricional. Debe ser una estrategia multidisciplinaria, que debe implementarse ya desde niños, pues si bien la educación nutricional se asocia a la conducta alimentaria, solo podría ser efectiva si la duración y frecuencia son adecuadas y reforzadas en las casas a través del ejemplo de los familiares. Los resultados de la presente investigación podrían suponer que la implementación de las guías alimentarias del Paraguay, como actualmente se están implementado, no son una estrategia efectiva para superar la malnutrición y las enfermedades crónicas relacionadas a la dieta que aquejan a las sociedades modernas, por el alto mantenimiento de estilos de vida inadecuados y la falta de interés en cambiarlos, a excepción del lavado y manipulación de alimentos.

\section{REFERENCIAS BIBLIOGRÁFICAS}

1. López Nomdedeu C, coordinadora. Nutrición saludable y prevención de los trastornos alimentarios. /Internet/: Ministerios del Interior, Ministerio de Educación y Cultura, Ministerio de Sanidad y Consumo. /citado 19 nov 2014/. Disponible en: http://www.fhspereclaver.org/userfiles/file/guia_nutricion_saludable.pdf

2. Kohen V, Gómez C, Lourenço T, Castillo R, Huerta M, Zurita L. Evaluación de la utilidad de un programa de educación nutricional en trastornos de la conducta alimentaria. Nutr Clin Diet Hosp. 2007; 27(3): 7-17.

3. Angeleri M, González I, Ghioldi M, Petrelli L. Educación alimentaria nutricional (EAN) en colegios: Evaluación de la efectividad de una intervención educativa. DIAETA. 2008; 26 (125):6-11.

4. López MA. Etapas del cambio conductual ante la ingesta de frutas y verduras, control de peso y ejercicio físico de estudiantes de la universidad del desarrollo, sede Concepción, Chile. Rev Chil Nutr. 2008; 35(3):215-24.

5. Landaeta-Jimenez M, Patiño E, Galicia N. Educación nutricional y participación; claves del éxito en la nutrición comunitaria. An Venez Nutr. 2005; 18(1):134-7.

6. Instituto Nacional de Alimentación y Nutrición (INAN). Guías alimentarias del Paraguay. Asunción. Dirección General de Programas de Salud, Ministerios de Salud Pública y Bienestar Social; 2010.

7. Molina V. Guías alimentarias en América Latina: Informe de la consulta técnica regional de las guías alimentarias. ALAN. 2008; 21(1):31-41.

8. Olivares S, Lera L, Bustos N. Etapas del cambio, beneficios y barreras en actividad física y consumo de frutas y verduras en estudiantes universitarios de Santiago de Chile. Rev chil nutr. $2008 ; 35(1): 25-35$.

9. Organización Mundial de la Salud. Enfermedades no trasmisibles. Ginebra: OMS; 2013.

10. Cabrera G. Etapas de Cambio en consumidores de cigarrillos de Zarzal, Colombia 1999. Rev. Fac. Nac. Salud Pública. 2001; 19(2):33-42.

11. Cañete F. Primera encuesta nacional sobre factores de riesgo y enfermedades no transmisibles. /Internet/. Asunción: Ministerio de Salud Pública y Bienestar Social, Dirección de Encuestas, Estadísticas y Censos; 2011 /citado 19 nov 2014/. Disponible en: http://www.nutrisyspy.com/descarga/img/-ENT.pdf 
12. Gutiérrez-Fisac, JL. Indicadores de consumo de alcohol en España. Med Clin (Barc). 1995; 104: 544-50.

13. Cabrera C G, Gómez L, Mateus JC. Actividad física y etapa de cambio comportamental en Bogotá. Colomb Méd. 2004; 35(2):82-6.

14. Ministerio de Salud Pública y Bienestar Social. Guías Alimentarias del Paraguay se actualizan después de 13 años. Asunción: MSPBS; 2013. 